\title{
Energy Efficiency Improvement through Optimal Batch Sizing in Job Shop
}

\author{
Samira Alvandi ${ }^{1}$ \\ ${ }^{1}$ School of Engineering and Technology, Central Queensland University, Sydney, Australia \\ Correspondence: Samira Alvandi, School of Engineering and Technology, Central Queensland University, \\ Sydney, Australia.
}

Received: December 19, 2018

Accepted: September 14, 2020

Online Published: September 21, 2020

doi:10.5539/mas.v14n10p6

URL: https://doi.org/10.5539/mas.v14n10p6

\begin{abstract}
The increasing customization of products with greater variances and smaller lot sizes, has motivated manufacturers to adopt highly dynamic production planning. The production plans not only need to adapt to the production system state changes rapidly but also need to adopt energy reduction schemes to satisfy key sustainability performance indicators. The dilemma from industry point of view is to tackle multi-faceted problem of optimising economic and environmental performance. This research aims to overcome the multi-faceted objectives of small and medium-sized enterprises (SME's) by providing a simulation-optimisation platform that creates the best possible production plans for optimum results. The applicability of the proposed framework is demonstrated through a real-life job-shop environment with the focus on optimisation of energy as well as job tardiness.
\end{abstract}

Keywords: production planning, simulation-optimisation, optimal batch sizing, manufacturing systems

\section{Introduction}

As the world becomes more populous and urbanised, the demand for commodities to satisfy the economic growth has dramatically increased. Resource scarcity and depletion of raw material pose significant challenges for manufacturing globally. It is estimated that, there may be just half a century's worth of oil and gas left at current rates of consumption (Jones et al., 2013). This growing problem has triggered many initiatives around the world to address the challenges of producing low cost and low environmental impact products.

Factories are known as complex systems consisting of production machines, supporting (auxiliary) services that ensure suitable environment such as temperature, ventilation, compressed air for manufacturing of the products. All the production machines and auxiliary supports (Technical Building Services- known as TBS) are complex, dynamic systems which consume resources such as energy, water, and raw materials. The inefficient consumption of resources contributes greatly to the economic and environmental well-being of these systems. Adaptation of efficient technologies, application of resource conscious production through multi-objective production planning (scheduling), are just few examples of many other strategies to improve and maximise economic and environmental position of the businesses.

Manufacturing systems are subject to dynamic influences from within the factory; unforeseen changes to the sequence of orders, machine breakdowns and customisation of products, are just few examples of such dynamic and ever-changing environment. As production moves from mass customization towards individualized or customised production with each product having different work steps and cycle times, the static cycle times as well as batch size of assembly lines do not satisfy the scheduling task and production plans anymore. Also, recognising the fact that Production schedule and product routings directly affect the production lead times and total energy consumed by manufacturing systems (Alvandi et al., 2016), traditional practices of production planning fail to tackle the challenges of current industrial and business needs.

This paper introduces a holistic approach for multi-objective optimisation, considering energy and traditional business objectives aiming at maintaining optimal utilization of machines, throughput and energy usage and producing different product ranges with high variance in lead times. 


\section{Background}

\subsection{Optimized Scheduling of Manufacturing Systems}

To deal with real-world complex systems, it is vital to utilise simulation to evaluate the system's performance for each set of decision variables (Longo, 2010) and (Lin and Chen 2015). However optimal decision making for complex and stochastic systems, such as the job-shop scheduling, is something that cannot be achieved through simulation alone.

A promising approach is by combining simulation with optimization. The idea of combining optimization and simulation has been studied since the beginning of this millennium (Fu, 2002). Hybrid simulation-optimisation problems usually deal with uncertainty, non-linear relationship, qualitative aspects, or even deal with processes that can be hardly modelled by analytical expressions (Figueira \& Almada-Lobo, 2014).

In this setting, the simulation model is used as the objective function of the optimization and the optimization method determines the optimal configuration of parameters for the simulation (Krug et al., 2002). Gupta and Sivakumar (2002) optimised scheduling of jobs on a single machine. The objectives were to minimise average tardiness and average completion time and maximisation of machine utilisation. Liberopoulos and Koukoumialos (2005) used an approach of simulation-based optimization in a make-to-stock environment to study the trade-offs between base stock levels, numbers of Kanban, and planned supply lead times. Arakawa, Fuyuki, and Inoue (2003) solved the operational planning problem of job shop scheduling using an optimization in conjunction with simulation. Kämpf and Köchel (2006) focused on sequencing and lot sizing problem in production-inventory system and applied simulation-based optimization model method.

Ehrenberg and Zimmermann (2012) proposed a simulation-based optimization approach to determine schedules for a make-to-order production of companies that manufacture special purpose glasses. Melouk at al. (2013) created a decision support model for steel manufacturing and tested the impact of simultaneous change of inventory levels. For optimization they link their simulation model with an optimization module using OptQuest. The objective of their study was minimisation of costs arising from both inventory holding and process downtime. Kulkarni and Venkateswaran (2015) presented a hybrid modelling approach and used simulation-based optimisation for solving job shop scheduling. The chosen decision variables were delays and queue priorities in a job shop environment of three machines and three jobs.

\subsection{Energy Efficiency Evaluation and Optimization}

To solve multi-objective problems in the realm of job shop scheduling, it is necessary to consider the stochastic interrelations between machine job orders containing different products and resulting lead times, energy demands and peaks on factory level (including auxiliary processes and TBS). In a typical manufacturing system, there are many variables that affect the product flow and energy consumption. These variables can be linked with a single machine or a process chain, or the whole factory (Herrmann and Thiede, 2009).

In terms of energy efficiency evaluation, most approaches carried out what-if scenario assessments to find the impact of certain variables on the performance of the manufacturing systems (Sproedt et al., 2015; Schönemann et al., 2014; Dietmair et al., 2011). Only limited approaches are found that tackled multi-variable optimisation problems. Greenwood et al. (2005) presented a decision support tool for production scheduling at a ship panel shop. Incoming orders were simulated in sequences based on a set of common dispatching orders (e.g., shortest processing time). The study resulted in optimum performing sequence with respect to a weighted multi-criteria objective.

Fang et al. (2011) developed a multi-objective model for optimizing production schedule of a flow shop. The objective functions for optimisation study in this work were productivity (make span) and energy related performance indicators such as energy consumption, carbon footprint and peak power load. The multi-objective model considered scheduling problem of 36 jobs on two machines. A Pareto frontier was developed to show the trade-off between throughput time and peak power. Although this work is of a particular interest in relation to energy optimisation studies in production planning, the applicability of this method on a real-life job shop environment that encompass hundreds of jobs flowing through large number of machine tools, is not validated.

Another work by Mouzon, Yildirim, and Twomey (2007) studied the problem of scheduling of a CNC machine in order to reduce energy consumption and total completion time. The result showed $80 \%$ energy saving was possible by turning off non-bottleneck machines or putting the machines on idle mode. The underlying shortcoming of this work is, it only considered a single machine as decision variable tackling a single objective problem. Hence multi-variability and multi-objectivity were not considered.

Mouzon \& Yildirim (2008) suggested multi-objective optimization to minimize total energy consumption and 
total tardiness of one machine. Thiede (2011) and (Mousavi et al., 2015) explored the benefits of simulation techniques for assessing the dynamic energy consumption within the overall system. Mousavi et al. (2015) used simulation modelling to overcome some of the limitations in literature in terms of dynamics of individual processes and the entire system with the TBS demand management. They studied scheduling problems with five different products on the mass production and job shop environment. The shortcomings of this study is that the optimisation study only considered eight machines and did not consider the range of attributes (variables) such as complex job sharing, product routings and random flows, focusing merely on sequential flow in a form of continuous production.

Rodrigues et al. (2018) proposed a method of analysing electrical energy consumption using discrete event simulation integrated with optimisation through OptQuest module. The study aimed to evaluate the impact of changes on process parameters such as machine idle time and change over on sustainability indicators. However, the presented conceptual model is not validated and the results of the two case studies are yet to be published as future work as indicated by the authors. Moreover, the authors also specified the optimisation module in-use required a large number of simulation runs, such exercise can adversely affect the applicability of the model.

In realm of simultaneous optimisation of manufacturing systems, a few presented models lack validation which undermines the accuracy of the methodology as well as applicability to industry. Emphasis has been predominately on operational decision-making tools rather than energy-oriented optimisation.

To address the gap in literature, the focus of this paper is on energy-oriented optimisation of job shop through simulation using the integrated simulation-optimisation framework presented and published earlier (Alvandi et al., 2017). To this end, a real-life job shop industrial facility is chosen, involving more than 100 different job orders for variety of product types passing through multiple routes. In the following section, the proposed integrated framework is presented.

\section{The Integrated Simulation-Optimisation Framework}

The proposed simulation-optimisation is comprised of simulation model as the first segment and the optimisation engine (optimiser) as the second segment (see Figure 1).

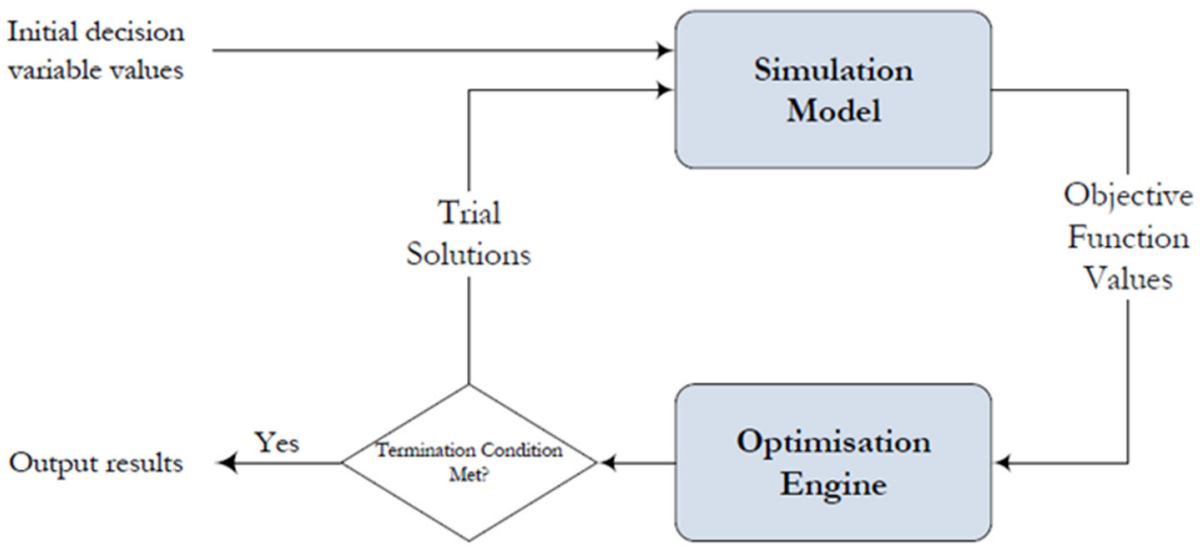

Figure 1. Integrated Framework of Simulation-based Optimisation (Alvandi et al., 2017)

A simulation model and an optimisation engine work in tandem and exchange information. The simulation model represents the manufacturing system and consists of the physical components of the system and existing logic.

Within the simulation model, the flow of material, information, and different types of energy are presented. The optimisation engine uses search algorithm and seeks optimal or near optimal solution(s) through searching the solutions space (one set of solution of solutions at a time).

The simulation model adopts a modular concept using Anylogic simulation software package by XjTek using a subset of UML for Real Time (UML-RT) as a modelling language platform. The outline of the conceptual framework of the simulation model is presented in Figure 2. 


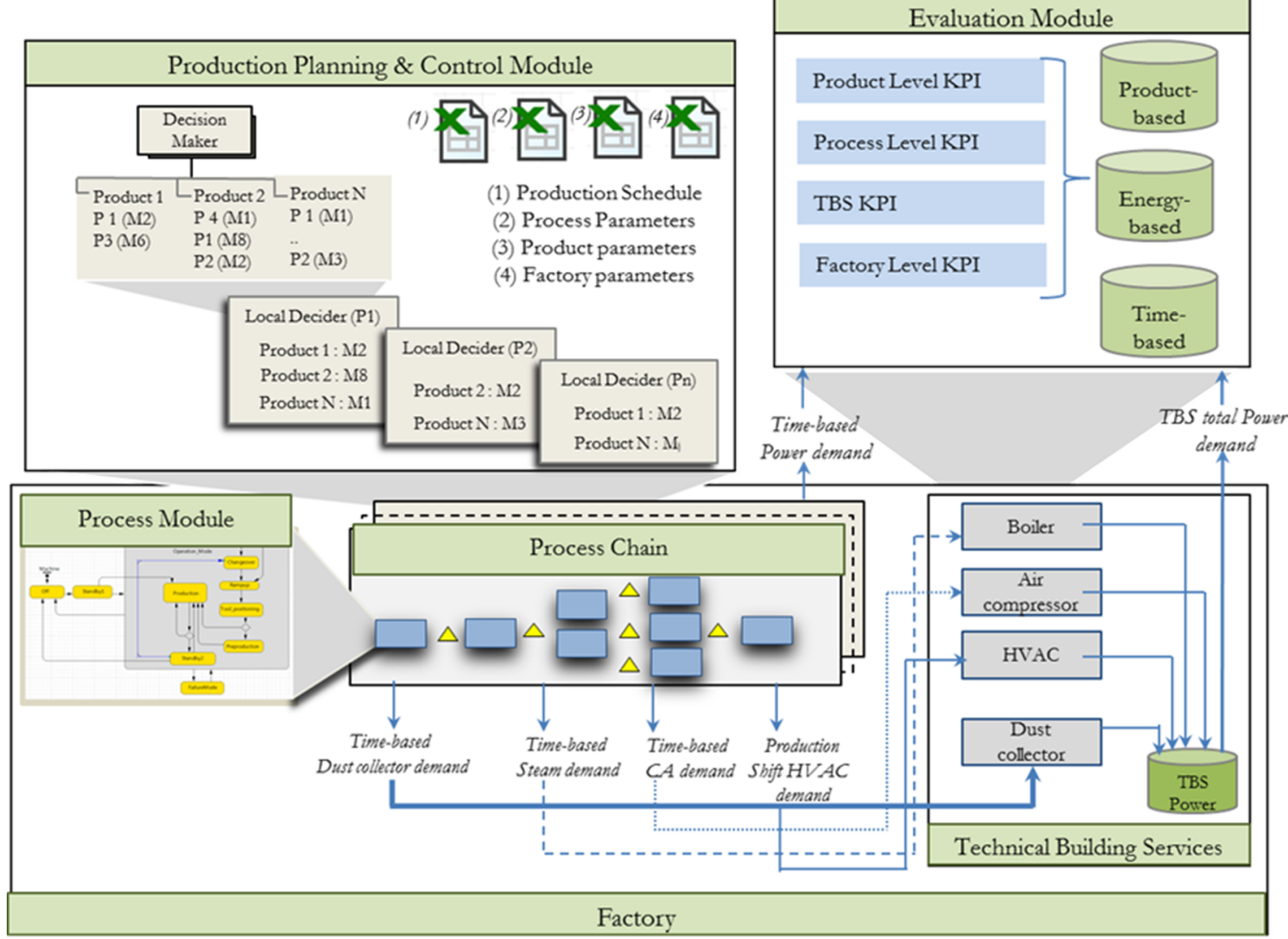

Figure 2. Conceptual framework of the simulation model (adopted from (Herrmann et al., 2011))

A process chain is configured by linking the process modules (machines) which are responsible to transform the material into the valuable product. Process module is modelled using hybrid modelling technique which combines queue theory and the state-chart models. The dynamic flow of energy within processes (machines) is approximated by configuring the states. The definition of each state of the machine is described in Table 1.

In most manufacturing systems the production processes must be powered by resources such as compressed air or heat that is mainly provided by TBSs. In this framework these supplied services include steam, compressed air, and conditioned air, cooled air and air purification. Two modules were developed to model cooling towers and dust collectors and three modules were adopted and considered in this framework to model steam generation units, compressed air systems and HVAC units (Ghadimi et al., 2014) and (Mousavi et al., 2014).

The production planning and control module (PPCM) is consist of four kinds of information sheets, as listed below.

- $\quad$ Production Schedule Module

- $\quad$ Process-Specific Parameter Sheet

- $\quad$ Product-Specific Parameter Sheet

- $\quad$ Factory-Level Parameter Sheet

The actual weekly production schedule is stored in the Excel sheet format and is interfaced with the simulation model via connectivity pallet of Anylogic. A production schedule is composed of the production plans for several production lines. Within this spread sheet the quantity of each product and sequences of the production is determined and fed to the system. 
Table 1. Various Machine States

\begin{tabular}{ll}
\hline Machine State & Definition \\
\hline Off & the state when the machine is switched off at the end of the production shift. \\
Ramp up & $\begin{array}{l}\text { production; normally results in a power surge. } \\
\text { the period when the machine remains ready for production. }\end{array}$ \\
Standby & the period when the positioning of the machine tool on the work piece is performed. \\
Tool Positioning & the state when the process (e.g. extrusion) needs adjustment and pilot runs are \\
Pre-Production & performed to verify the quality of the output. \\
& the state when the machine is processing the material. \\
Production & the state when the machine is being set up for producing a different product. \\
Changeover & refers to the state that the machine breaks down and requires repairs or maintenance. \\
Failure &
\end{tabular}

The second group of information (Process-Specific parameters) refers to the values required to set individual process modules, such as: transition times, machine states, energy requirement values of each state, scrap rate, mean time between failure (MTBF) and mean time to repair (MTTR).

Product-Specific information includes general information related to each product type such as process routing, product-specific batch size, and product-specific processing time. Multi-Product Routing module imports data from Job Matrix Excel spread sheet. The routing of each product consists of two parts: Process number and Machine number. Products can flow through consecutive processes. The product flow is also arbitrarily and does not necessarily be in a sequential manner.

The fourth group refers to the values required to set the steam generation module, the compressed air module, and the HVAC modules. At the time of running simulation all relevant variables are transferred to the evaluation module where key figures and tables are generated. Hence within evaluation module all the segregated analysis on product, process and system level are performed and visualised.

\subsection{Problem Definition}

As a proof of concept, the methodology outlined above is applied to a company that manufactures various products for the electricity distribution network. The manufacturing company is a typical job-shop factory with high product variations and small batch sizes. This job shop consists of variety of equipment and machinery such as drills, lathes, saws and flexible machines such as CNC lathe. As for the product flow between machines, it is not necessary for all operations and activities to be performed on all products. The work piece (product) is only passed to those machines that are required as per the product routing. As a result, the sequences of operations and number of operations on products vary a great deal.

Product mix in this factory varies from week to week, even day to day, and volumes fluctuate widely. To cater for all this, the utilisation of the general-purpose machines relies greatly on highly skilled labour. Machines must be reconfigured, and work orders must be rescheduled to meet customer timetables and specifications. Production runs 5 days per week with two 8-hr shifts per day. In terms of IT infrastructure, only basic ERP is available which updates the master production plan at a weekly basis. The production plan for one month is considered which involves around 32 different machines, each with different loading schedules. Products can be classified into 10 product family groups which signifies different process step requirement and different machine set up.

The whole factory is modelled by dragging and dropping of generic modules to the main simulation template. The unit processes represent each individual machine and are grouped under specific workstation. At this level, machine data are used to describe the behaviour of the available manufacturing processes. 


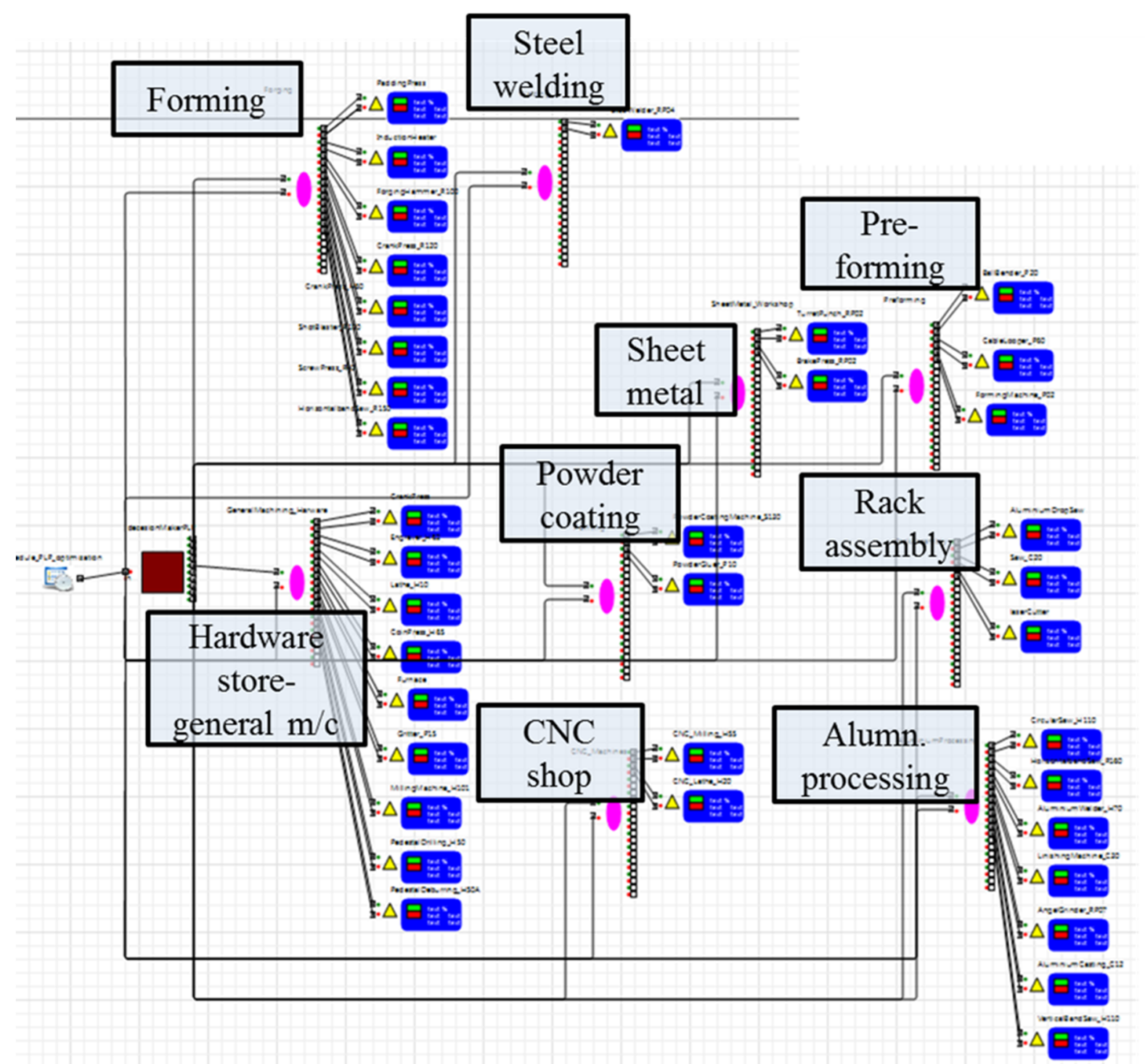

Figure 3. Snapshot of the simulation model of the job shop

In particular, machine-specific parameters (e.g. set-up and processing times, MTTR and MTBF) and machine energy profiles are highly relevant to derive the energy consumption. Depending on defined MTTR parameter, machine spends certain amount of time in Failure state before entering the Operation-state again. A Weibull distribution is used to mathematically describe the failure of the module, as in (Thiede, 2012). Figure 3 depicts a snapshot of simulation model with all the workstations and machine modules that were configured according to how they appear on the shop-floor.

A multi-objective optimisation module is connected with the simulation model to derive optimal settings for future operation. The primary goal of the optimisation module is to quantitatively analyse total energy consumption and job tardiness which will be described in more details in section 3.1. A classic method, the weighted sum, is used to form the multi-objective function due to its simplicity (see Equation 1).

$$
\begin{array}{ll}
\text { Minimis } & \mathrm{F}(\mathrm{X})=\sum_{k m=1}^{n m} W_{m} F_{m} \mathrm{~F}(\mathrm{X}) \\
& \mathrm{g}_{\mathrm{j}}(\mathrm{X}) \geq 0, \quad \mathrm{j}=1,2, \ldots, \mathrm{J} ; \\
& h_{k}(\mathrm{X}) \geq 0, \quad \mathrm{k}=1,2, \ldots, \mathrm{K} ; \\
& \mathrm{xi}^{(L)} \leq \mathrm{X}_{\mathrm{i}} \leq \mathrm{xi}^{(U)} \quad \mathrm{i}=1,2, \ldots, \mathrm{N} ;
\end{array}
$$

Equation 1 
Where $W_{m} \in[0,1]$ is the weight of the $\mathrm{m}^{\text {th }}$ objective function so that $\sum_{m=1}^{M} W_{m}=1 ; \mathrm{g}(\mathrm{X}), \mathrm{h}(\mathrm{X})$ refer to constraint functions; where $\mathrm{x}_{\mathrm{i}}^{\left({ }^{(L)}\right.}$ and $\mathrm{x}_{\mathrm{i}}{ }^{(U)}$ refer to the lower and upper limits of the $\mathrm{i}^{\text {th }}$ decision variable $\mathrm{xi}$.

To solve the multi objective problem, normalisation method is applied by transforming objective functions into single objective. The equations that are used for transformation of multi objective functions into single objective function are described in Equation 2 and Equation 3. For minimisation objectives Equation 2 is used and for maximisation objectives Equation 3 is applied.

$$
\begin{aligned}
& f_{i_{k}}^{(\text {trans })}=\frac{f_{i_{k}}(x)-f_{i_{k}}^{(\min )}}{f_{i_{k}}^{(\max )}-f_{i_{k}}^{(\min )}} \quad, 1 \leq i \leq N \\
& f_{i_{k}}^{(\text {trans })}=\frac{f_{i_{k}}^{(\max )}-f_{i_{k}}(x)}{f_{i_{k}}^{(\max )}-f_{i_{k}}^{(\min )}}, 1 \leq i \leq N
\end{aligned}
$$

Where $f_{i_{k}}(x), f_{i_{k}}^{(\min )}$ and $f_{i_{k}}^{(\max )}$ are current, the best and worst values of the objective function $k$ respectively for $1 \leq k \leq m$. Here the $f_{i_{k}}^{(\text {trans })}$ generally has values between zero and one, depending on the accuracy and method with which $f_{i_{k}}^{(\max )}$ and $f_{i_{k}}^{(\min )}$ were determined. In both equations, zero value had the highest utility.

\subsection{Problem Definition}

The job shop operates under imposing and conflicting parameters resulting in high operational inefficiency. The company's response to the stochastic demand for their products is to batch together many months' requirements of each product. The defined minimum batch sizes for each machine triggers the production to start. This causes unnecessary inventory to hold up and queuing times between successive operations since work on one order cannot begin until enough orders accumulate to form an adequate batch.

In order to reform the job shop in terms of its batching scheme and to optimise its performance, a multi objective optimisation problem is defined. The batch sizes for each of the product types have been studied and the ranges under which the batch sizes vary, are noted. The minimum and maximum batch size and step sizes are thus determined. The following table (Table 2) depicts the variable setting and ranges that were used in setting up the OptQuest parameters.

Table 2. Product-batch size ranges (Decision Variables)

\begin{tabular}{lllll}
\hline Parameter & Type & Min & Max & Step \\
\hline BatchSize_P1 & discrete & 100 & 800 & 100 \\
BatchSize_P2 & discrete & 25 & 200 & 25 \\
BatchSize_P3 & discrete & 100 & 600 & 100 \\
BatchSize_P4 & discrete & 50 & 200 & 50 \\
BatchSize_P5 & discrete & 500 & 3500 & 500 \\
BatchSize_P6 & discrete & 50 & 200 & 50 \\
BatchSize_P7 & discrete & 50 & 200 & 50 \\
BatchSize_P8 & discrete & 50 & 200 & 50
\end{tabular}

The minimum batch size that the company currently uses fall within the range and hence is one solution out of many available solutions. The aim is to find the best possible combination of product's batch sizes that result in optimum output of the system. For the purpose of this optimisation study, total tardiness and total energy consumption are considered as two objectives to be optimised simultaneously.

Let $\mathrm{Dj}, \mathrm{j} \in \mathrm{N}$, be the due date for the job $\mathrm{j}$, the job's tardiness is defined as $\mathrm{Tj}=\max \{\mathrm{Cj}-\mathrm{Dj}, 0\}$, where $\mathrm{Cj}$ is the completion time of job $\mathrm{j}$. The objective is to minimise total tardiness over all jobs, as illustrated in Equation : 


$$
T T=\sum_{j=1}^{n} T_{j}
$$

Total electrical energy consumption is calculated for the entire factory by aggregating the energy consumption of all the production machines as well as TBS and conforms to Equation 5:

$$
\text { E total }=\sum_{k=0}^{n} P_{k} d_{t}
$$

Where $\mathrm{k}$ refers to the $\mathrm{kth}$ process and TBS equipment; $\mathrm{P}_{\mathrm{k}}$ refers to the power demand of $\mathrm{k}^{\text {th }}$ process.

\subsection{Solving Multi-Objective Optimisation}

In selecting the optimisation method, one of the key characteristics is applicability of the method on different simulation-based models. Advances in the area of metaheuristic optimisation have provided researchers with great advantage in using off-the-shelf, general purpose optimiser packages.

OptQuest ${ }^{\mathrm{TM}}$ is one of these general-purpose commercial packages that was developed in the framework of a metaheuristic methodology (OptTek Systems, 2015). To guide the built-in search algorithm, OptQuest incorporates a combination of metaheuristics such as scatter search, tabu search and neural networks (Glover, Kelly, \& Laguna, 1996). The simulation model is integrated with optimisation engine by configuring four key elements: objective functions and their weights, decision variables, constraints, and stop condition. With this configuration, simulation model is used as the objective function for the optimization which in return determines the optimal configuration of input parameters for the simulation.

For this study, the production is simulated for eight days period. The simulation warm-up takes a short time in this case due to much shorter production times in relation to job orders. It takes one day worth of production for the system to reach a steady state. Therefore, one day warm up is discarded from the 8-day production and the objective functions are measured for seven days.

\subsubsection{Total Tardiness Minimisation}

The OptQuest is set to minimise total tardiness. After 249 runs, simulation-optimisation stopped indicating run number 163 as the best result for objective function. The detail of the result is presented in Table 3 .

Table 3. Objective values when minimising total tardiness

\begin{tabular}{cccc}
\hline Best Run & Total run & Total Tardiness $(\mathrm{min})$ & Energy $(\mathrm{kWh})$ \\
\hline 163 & 249 & $* 85057$ & 46060 \\
\hline *optimised values & & &
\end{tabular}

From the variable setting point of view, the optimum batch sizes recommended by OptQuest are shown as per Table 4.

Table 4. Optimum batch size, total tardiness minimised

\begin{tabular}{lc}
\hline Variable & Optimum Result \\
\hline BatchSize_P1 & 100 \\
BatchSize_P2 & 25 \\
BatchSize_P3 & 100 \\
BatchSize_P4 & 100 \\
BatchSize_P5 & 500 \\
BatchSize_P6 & 150 \\
BatchSize_P7 & 50 \\
BatchSize_P8 & 50 \\
\hline
\end{tabular}

In addition to optimising the total tardiness, the non-value added time for each process was also recorded for further analysis. Non-value adding time of the process is defined as the time that the machine is not processing the material such as in ramp up or changeover. The statistical analysis is performed on the non-value added time for the whole 249 runs (see Figure 4).

The aim is to see how sensitive each process is in relation to changes to the batch size. All the 34 processes 
(Y-axis) with their min, average, max values for their resulting non-value added time are plotted. The plotted diagram shows interesting results. As expected, the waiting on general purpose machines that process different types of products are longer than the single product machines.

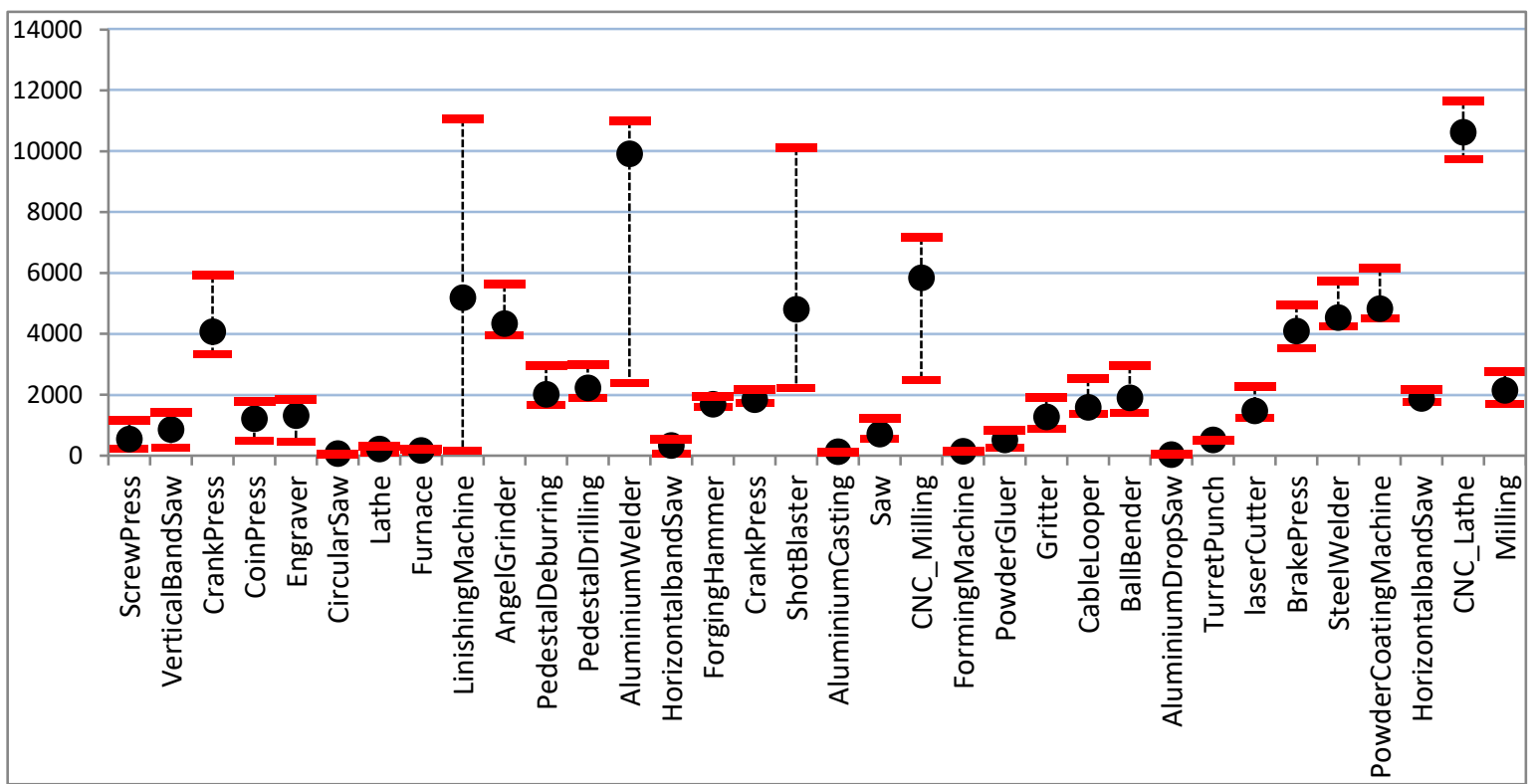

Figure 4. Statistical analysis on non-value added times of each machine, total tardiness minimised

The five largest variations in the non-value added times are related to the five processes that are mostly used within other product group's production lines. The list of the machines and the product types that share these machines are summarised below (see Table 5). There is a competition in using the Crank press, Finishing and $\mathrm{CNC}$ milling between product types. The product that reaches the batch size limit wins the machine time and gets in first for processing and obviously gets out earlier. By changing the batch sizes of the products on these highly utilised machines, the queue time is affected.

Table 5. List of shared-machines between product families

\begin{tabular}{ll}
\hline Machine & Product \\
\hline Crank Press & CTA, Shakle, Switch Gear \\
Finishing & CTAW (2times), Terminal Palm, Busbar, Switch Gear \\
AluminiumWelder & Busbar, Switch Gear \\
ShotBlaster & Shakle, Busbar \\
CNC_milling & Terminal Palm, Busbar, Switch Gear \\
\hline
\end{tabular}

Aluminium welder and Shot blaster are shared between fewer product types compare to other three machines, nevertheless they also show high level of sensitivity to changes of batch sizes. Looking at the production line for the corresponding products P6, P8 and P3, it is noted that Shot blaster and Aluminium Welder are located at the far end of the chain.

The upstream machines to this lower stream machines include the Crank press, Finishing and CNC mill. These three machines act like a bottleneck and slow down the product flow. Consequently, it is logical to see the lower stream machines get affected.

\subsubsection{Energy Minimisation}

Optimisation was run with the Energy as the main objective function to be minimised. The value of the objective function and also the value of total tardiness achieved at this optimum result are shown in Table 6.

Table 6. Objective values when minimising energy

\begin{tabular}{cccc}
\hline Best Run & Total run & Total Tardiness (min) & Energy $(\mathrm{kWh})$ \\
\hline 234 & 281 & 171258 & $* 41505$ \\
\hline *optimised values & &
\end{tabular}


The energy consumption at $41505 \mathrm{kWh}$ is the minimum possible value the company could achieve through choosing the combination of batch sizes as illustrated in Table 7 .

Now with Energy as an objective function for the optimisation problem, the sensitivity of the processes in terms of non-value added times is analysed through plotting the min, average, max non-value added times for all 34 processes (Screw press, Vertical Band Saw, etc.) as presented in Figure 5.

Table 7. Optimum batch size, energy minimised

\begin{tabular}{lc}
\hline Variable & Optimum Result \\
\hline BatchSize_P1 & 500 \\
BatchSize_P2 & 150 \\
BatchSize_P3 & 300 \\
BatchSize_P4 & 150 \\
BatchSize_P5 & 2000 \\
BatchSize_P6 & 100 \\
BatchSize_P7 & 150 \\
BatchSize_P8 & 150 \\
\hline
\end{tabular}

The five processes with high variation in the previous optimisation analysis, still showing the same trend. Although the average waiting for most of the processes differ from previous results, the non-value added times is clearly proved to be impacted by the batch size changes.

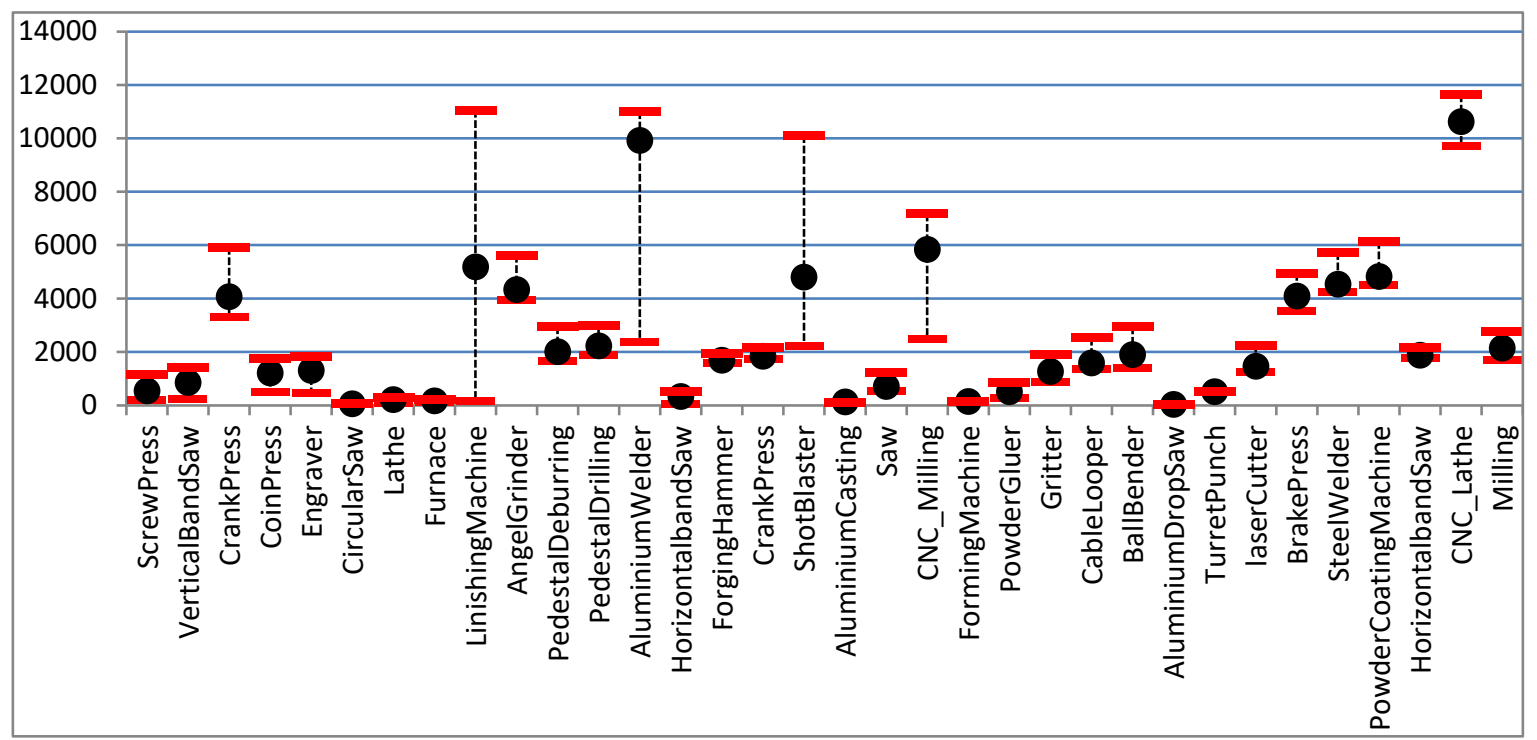

Figure 5. Statistical analysis on non-value added times of each machine, energy minimised

\subsubsection{Multi Objective Optimisation}

The proposed optimisation engine, as explained in section 3, utilises the weighted sum method to combine several objective functions. Table 8 presents the best and worst values of each objective function generated based on the single optimisation problems.

Table 8 . The best and the worst of each objective during single optimisation runs

\begin{tabular}{lccc}
\hline \multicolumn{2}{c}{ Primary objective function } & Energy $(\mathrm{kWh})$ & Total Tardiness (min) \\
\hline Energy & Min & $41505^{*}$ & 95388 \\
\multirow{4}{*}{ Total Tardiness } & Max & 52946 & 262266 \\
& Min & 41644 & $85057^{*}$ \\
& Max & 52742 & 263009 \\
\hline \multirow{5}{*}{ Min } & 41505 & 85057 \\
& Max & 52946 & 263009 \\
\hline
\end{tabular}


The $\min / \max$ values of objective functions are then used as an input. The weighted sum method needs the relative importance of each objective function or weights. Since simultaneous optimisation of all is the main goal, the equal weight of 0.5 has been given to both objectives. Table 9 presents the optimum results for multi-objective problem achieved at run number 217.

Table 9. Optimal value of objectives with equal importance

\begin{tabular}{ccccc}
\hline Objective function & Best Run & Total run & Total Tardiness & Energy \\
\hline WF(x) & 217 & 289 & 90563.36 & 41923.10 \\
\hline
\end{tabular}

\subsection{Sensitivity Analysis}

In this section, the sensitivity of the objective function weights was studied by considering three more scenarios in addition to two single objective scenarios. The relative importance of each objective function- $w 1$ and $w 2$ are apportioned to the problem case with two objectives.

As shown in the previous section, S0 was one of the extreme points for the optimisation problem in which full attention was given to total energy (Energy) by assigning the maximum possible weight of 1.00 to it and disregarding the total tardiness objective. S1 scenario on the other hand refers to optimisation of total tardiness objective where the Energy was not the focus of the optimisation problem.

Moreover, three more scenarios S2, S3 and S4 were designed. Table 10 summarises the weights of objectives in all five scenarios. The simulation model of scenario 2, 3, and 4 were optimised. The optimal values of the decision variables and objective functions are also provided in Table 10.

Table 10. Optimisation results for 5 scenarios

\begin{tabular}{ccccc}
\hline Scenario & $\begin{array}{c}\text { Energy } \\
(\text { weight })\end{array}$ & $\begin{array}{c}\text { Total tardiness } \\
(\text { weight })\end{array}$ & $\begin{array}{c}\text { Energy } \\
(\mathrm{kWh})\end{array}$ & $\begin{array}{c}\text { Total tardiness } \\
(\mathrm{min})\end{array}$ \\
\hline S0 & 100 & 0 & 41505.40 & 171258.86 \\
S1 & 0 & 100 & 46060.22 & 85057.89 \\
S2 & 0.5 & 0.5 & 41923.10 & 90563.36 \\
S3 & 0.3 & 0.7 & 41950.43 & 98033.33 \\
S4 & 0.7 & 0.3 & 41564.20 & 135762.41 \\
\hline
\end{tabular}

The results agree with those achieved under single objective optimisation exercises, which also suggest that the batch size has a greater impact on the job tardiness than on the energy consumption. To explain this, the solutions were studied carefully. The study revealed that although smaller batch sizes will reduce the queue times and job tardiness, it will increase the energy consumption as shown in Figure 6.

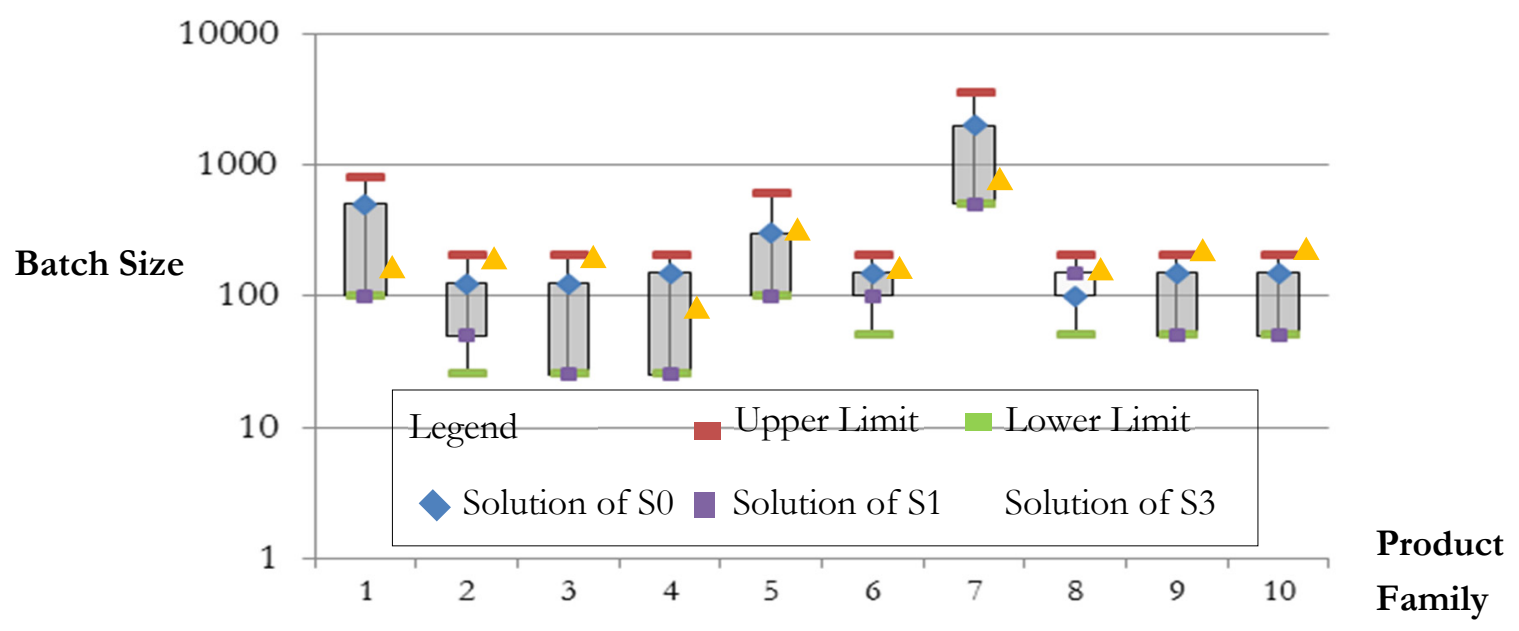

Figure 1. Optimisation solution - best set of batch sizes for product types

This outcome is mainly as a result of machine's frequent changeovers. There are higher number of change overs when using small batch size compare to larger batch size which corresponds to higher energy consumption during machine set up. 
Further comparison analysis of different scenarios with the baseline (i.e. scenario BS) in terms of objective functions was performed. Table 11 summarises the results of each scenario as well as the absolute and relative deviation between the baseline and other scenarios.

Comparing the baseline scenario (BS) with scenario S2 where the objective functions were given equal importance, revealed that around 7\% less Energy and 38\% improvement on total tardiness were obtained with the optimal solution. It should be noted that the maximum savings of $8 \%$ on Energy can be achieved when the focus of the optimisation is only on Energy.

Table 11. Absolute and relative deviation between baseline and other scenarios

\begin{tabular}{ccccccc}
\hline & & & \multicolumn{2}{c}{ Absolute deviation } & \multicolumn{2}{c}{ Relative deviation } \\
\hline \multirow{2}{*}{ Energy } & TT & Energy & TT & Energy & TT \\
& $(\mathrm{kWh})$ & $(\mathrm{min})$ & $(\mathrm{kWh})$ & $(\mathrm{min})$ & $(\mathrm{kWh})$ & $(\mathrm{min})$ \\
\hline BS & 44917 & 146273 & - & - & - & - \\
S0 & 41506 & 171259 & 3411 & -24986 & $8 \%$ & $-17 \%$ \\
S1 & 46060 & 85058 & -1143 & 61215 & $-3 \%$ & $42 \%$ \\
S2 & 41923 & 90563 & 2994 & 55710 & $7 \%$ & $38 \%$ \\
S3 & 41950 & 98033 & 2967 & 48240 & $7 \%$ & $33 \%$ \\
S4 & 41564 & 135762 & 3353 & 10511 & $7 \%$ & $7 \%$ \\
\hline
\end{tabular}

The distribution of scenario S0 to S4 shows the sensitivity of the optimisation process to the objective weights when a value greater than $70 \%$ was used for one objective. This was true for both objective functions. For example, when scenario S1 was compared with S3 (a 30\% reduction in the total tardiness weight) the total tardiness objective was worsened by around 15\% (from 85058 minutes in S1 to 98033 minutes in S3).

\section{Conclusion and Outlook}

Reducing electricity consumption as well as keeping good performance in classical job shop scheduling is a difficult problem that can take a large amount of time to optimally solve. From academic point of view, most of the current energy-conscious scheduling researches are single machine and flow shop oriented, while from practical point of view, majority of the factories are small and medium enterprises (SMEs) which operate in a job shop environment.

Addressing the existing gap in realm of energy efficient scheduling for a typical job shop, the proposed integrated simulation-optimisation framework was applied on a make-to-order company that supplies telecommunication parts. For the optimisation study, Product Batch size was considered as a controllable decision variable where eight products were selected with varying batch sizes. The eight variables were then varied from a lower bound to an upper bound on the batch size of each product. Total energy consumed by the system and total tardiness were used as objective functions and a weighted sum method was used to combine the objectives. Analysis of the optimisation results on total energy consumed by the system and total tardiness, revealed that there was a direct relationship between the two objectives. The results revealed that around $7 \%$ savings on energy and 38\% improvement on total tardiness are possible when both objectives are given equal importance for a simultaneous improvement.

In recognition of the multi-criteria optimisation within manufacturing systems, the opportunity exists to expand the proposed approach to include other sustainability key performance indicators (KPIs) such as Green House Gas $(\mathrm{GHH})$ emissions and waste generation for the entire product life cycle. Owing to black-box nature of the optimisation engine, the presented simulation-optimisation framework can be applied to any industry with any type of production system in-use. Further work will focus on inclusion of some environmental KPIs to be used strictly for the optimisation study.

Furthermore, with an increase in automation and intelligent systems (Radziwon et al., 2014), manufacturing industry need to adopt new approaches on optimization of their processes. One promising approach is the use of digital twin in obtaining a near-real-time production and operation data through sensors (Schroeder et al., 2016). During modelling, the processes can be replicated with their corresponding real time data rather than inputs from manufacturing database or assumptions, an exercise that results in a greater simulation accuracy for analysing manufacturing system performance and optimisation. 


\section{Acknowledgement}

The author would like to acknowledge Australian Research Council (ARC) and the Sustainable Manufacturing \& Life Cycle Engineering Research Group of the Mechanical \& Manufacturing Engineering at the University of New South Wales for supporting this research project.

\section{References}

Alvandi, S., Li, W., \& Kara, S. (2017). An Integrated Simulation Optimisation Decision Support Tool for Multi-Product Production Systems. Modern Applied Science, 11(6), 56. https://doi.org/10.5539/mas.v11n6p56

Alvandi, S., Li, W., Schönemann, M., Kara, S., \& Herrmann, C. (2016). Economic and environmental value stream map (E2VSM) simulation for multi-product manufacturing systems. International Journal of Sustainable Engineering, 1-9. https://doi.org/10.1080/19397038.2016.1161095

Arakawa, M., Fuyuki, M., \& Inoue, I. (2003). An optimization-oriented method for simulation-based job shop scheduling incorporating capacity adjustment function. International Journal of Production Economics, 85(3), 359-369. https://doi.org/10.1016/S0925-5273(03)00122-1

Dietmair, A., Verl, A., \& Eberspaecher, P. (2011). Model-based energy consumption optimisation in manufacturing system and macine control. International Journal of Manufacturing Research, 6(4), 380-401. https://doi.org/10.1504/IJMR.2011.043238

Ehrenberg, C., \& Zimmermann, J. (2012, 9-12 Dec. 2012). Simulation-based optimization in make-to-order production: Scheduling for a special-purpose glass manufacturer. Paper presented at the Proceedings of the 2012 Winter Simulation Conference (WSC). Berlin, 2012, 1-12. https://doi.org/10.1109/WSC.2012.6465047

Fang, K., Uhan, N., Zhao, F., \& Sutherland, J. W. (2011). A new approach to scheduling in manufacturing for power consumption and carbon footprint reduction. Journal of Manufacturing Systems, 30(4), 234-240. https://doi.org/10.1016/j.jmsy.2011.08.004

Figueira, G., \& Almada-Lobo, B. (2014). Hybrid simulation-optimization methods: A taxonomy and discussion. Simulation Modelling Practice and Theory, 46, 118-134. https://doi.org/10.1016/j.simpat.2014.03.007

Fu, M. C. (2002). Optimization for simulation: Theory vs. practice. INFORMS Journal on Computing, 14(3), 192-215. https://doi.org/10.1287/ijoc.14.3.192.113

Ghadimi, P., Kara, S., \& Kornfeld, B. (2014, 2-6 June 2014). Reactive modelling of on-site energy system components for real-time application. Paper presented at the Intelligent Energy and Power Systems (IEPS), 2014 IEEE International Conference. Kiev, 2014, pp. 219-224. https://doi.org/10.1109/IEPS.2014.6874183

Glover, Fred, James P. Kelly, \& Manuel Laguna. New advances and applications of combining simulation and optimization. Proceedings of the 28th conference on Winter simulation. IEEE Computer Society, 144-152, Coronado, California, USA, 1996. https://doi.org/10.1145/256562.256595

Greenwood, A. G., Vanguri, S., Eksioglu, B., Jain, P., Hill, T. W., Miller, J. W., \& Walden, C. T. (2005, 4-4 Dec. 2005). Simulation optimization decision support system for ship panel shop operations. Proceedings of the Winter Simulation Conference. 2005, Orlando, FL, 2005, https://doi.org/10.1109/WSC.2005.1574490

Gupta, A. K., \& Sivakumar, A. L. (2002). Semiconductor manufacturing: simulation based multi objective schedule optimization in semiconductor manufacturing. Proceedings of the 34th conference on Winter simulation: exploring new frontiers, San Diego, California, 2002. https://doi.org/10.1109/WSC.2002.1166480

Herrmann, C., \& Thiede, S. (2009). Process chain simulation to foster energy efficiency in manufacturing. CIRP Journal of Manufacturing Science and Technology, 1(4), 221-229. https://doi.org/10.1016/j.cirpj.2009.06.005

Herrmann, C., Thiede, S., Kara, S., \& Hesselbach, J. (2011). Energy oriented simulation of manufacturing systems - Concept and application. CIRP Annals - Manufacturing Technology, 60(1), 45-48. https://doi.org/10.1016/j.cirp.2011.03.127

Jones, A., Allen, I., Silver, N., Cameron, C., Howarth, C., \& Caldecott, B. (2013). Resource constraints: sharing a finite world Implications of Limits to Growth for the Actuarial Profession. Retrieved from https://www.actuaries.org.uk/documents/research-report-resource-constraints-sharing-finite-world-implicati ons-limits-growth

Kämpf, M., \& Köchel, P. (2006). Simulation-based sequencing and lot size optimisation for a production-and-inventory system with multiple items. International Journal of Production Economics, 104(1), 191-200. https://doi.org/10.1016/j.ijpe.2006.02.008 
Krug, W., T. Wiedemann, J. Liebelt, \& B. Baumbach. (2002). Simulation and Optimization in Manufacturing, Organization and Logitics. In Proceedings 14th European Simulation Symposium, edited by A. Verbraeck, and W. Krug, 7 pages. SCS Europe BVBA.

Kulkarni, K., \& Venkateswaran, J. (2015). Hybrid approach using simulation-based optimisation for job shop scheduling problems. Journal of of Simulation. https://doi.org/10.1057/jos.2014.40

Liberopoulos, G., \& Koukoumialos, S. (2005). Tradeoffs between base stock levels, numbers of kanbans, and planned supply lead times in production/inventory systems with advance demand information. International Journal of Production Economics, 96(2), 213-232. https://doi.org/10.1016/j.ijpe.2004.04.006

Lin, J. T., \& C. M. Chen. (2015). Simulation Optimization Approach for Hybrid Flow Shop Scheduling Problem in Semiconductor Back-End Manufacturing. Simulation Modelling Practice and Theory, 51, 100-114. https://doi.org/10.1016/j.simpat.2014.10.008

Longo, F. (2010). Emergency Simulation: State of the Art and Future Research Guidelines. SCS M \& S Magazine 1:2010-04.

Melouk, S. H., Freeman, N. K., Miller, D., \& Dunning, M. (2013). Simulation optimization-based decision support tool for steel manufacturing. International Journal of Production Economics, 141(1), 269-276. https://doi.org/10.1016/j.ijpe.2012.08.001

Mousavi, S., Kara, S., \& Kornfeld, B. (2014). Energy Efficiency of Compressed Air Systems. Procedia CIRP, 15, 313-318. https://doi.org/10.1016/j.procir.2014.06.026

Mousavi, S., Thiede, S., Li, W., Kara, S., \& Herrmann, C. (2015). An Integrated Approach for Improving Energy Efficiency of Manufacturing Process Chain. International Journal of Sustainable Engineering, 9(1), 11-24. https://doi.org/10.1080/19397038.2014.1001470

Mouzon, G., \& Yildirim, M. B. (2008). A framework to minimise total energy consumption and total tardiness on a single machine. International Journal of Sustainable Engineering, 1(2), 105-116. https://doi.org/10.1080/19397030802257236

Mouzon, G., Yildirim, M. B., \& Twomey, J. (2007). Operational methods for minimization of energy consumption of manufacturing equipment. International Journal of Production Research, 45(18-19), 4247-4271. https://doi.org/10.1080/00207540701450013

OptTek Systems, I. (2015). http://www.opttek.com/partners. Accessed Sep.2017

Radziwon, A., Bilberg, A., Bogers, M., \& Madsen, E. S. (2014). The smart factory: exploring adaptive and flexible manufacturing solutions. Procedia Engineering, 69, 1184-1190. https://doi.org/10.1016/j.proeng.2014.03.108

Rodrigues, G. S., Espíndola Ferreira, J. C., \& Rocha, C. R. (2018). A novel method for analysis and optimization of electric energy consumption in manufacturing processes. Procedia Manufactuing, 17, 1073-1081. https://doi.org/10.1016/j.promfg.2018.10.078

Schönemann, M., Thiede, S., \& Herrmann, C. (2014). Integrating Product Characteristics into Extended Value Stream Modeling. Procedia CIRP, 17, 368-373. https://doi.org/10.1016/j.procir.2014.01.091

Schroeder, G. N., Steinmetz, C., Pereira, C. E., \& Espindola, D. B. (2016). Digital twin data modeling with automation ML and a communication methodology for data exchange. IFAC Papers on Line, 49(30), 12-7. https://doi.org/10.1016/j.ifacol.2016.11.115

Sproedt, A., Plehn, J., Schönsleben, P., \& Herrmann, C. (2015). A simulation-based decision support for eco-efficiency improvements in production systems. Journal of Cleaner Production, 105, 389-405. https://doi.org/10.1016/j.jclepro.2014.12.082

Thiede, S. (2011). Energy Efficiency in Manufacturing Systems. (PhD Dissertation), Technische Universität Braunschweig, Germany. Retrieved from http://www.iwf.tu-bs.de/info/personen/SThiede.html

Thiede, S. (2012). Energy Efficiency in Manufacturing Systems: Springer-Verlang Berlin Heidelberg.

\section{Copyrights}

Copyright for this article is retained by the author(s), with first publication rights granted to the journal.

This is an open-access article distributed under the terms and conditions of the Creative Commons Attribution license (http://creativecommons.org/licenses/by/4.0/). 